J Nutri Health

September 2017 Vol.:3, Issue:2

(C) All rights are reserved by Agarwal, et al.

\section{Antioxidants-the Miraculous Molecules}

\section{Short Communication}

Nature is well equipped with the defense mechanisms to protect the living beings. Breathing is an inevitable process of life involving oxygen to produce ATP, biological currency of energy via electron transport chain in mitochondria of cells. Oxidation occurs during this process and some unpaired electrons are escaped which are called Free Radicals (FR). Latter are devoid of oxygen. Some free radicals contain oxygen and are referred as Reactive Oxygen Species (ROS). Under normal conditions generation of free radicals is part of energy metabolism and is used for the production of some hormones; normal cell signaling and engulfing pathogens. Since both FR and ROS are highly reactive molecules, they soon start chain reaction with biological molecules like lipids, proteins and DNA and alter them to gain electron, if not terminated or neutralized by antioxidants they become havoc for life and triggers range of human diseases.

When ROS cause damage to the cells and cellular components or their functionality and the condition is termed as oxidative stress. Oxidative stress is implicated in pathophysiology of acute or chronic diseases such as immunological disorders, infectious diseases, inflammatory disorders, cardiac disorders, neurodegeneration or neurological disorders, cataract, arthritis, chronic fatigue and some cancers. It accelerates the aging process itself.

Poor dietary habits, sedentary life style, exposure to UV rays, ionizing radiation, pollution, smoking, herbicides, pesticides, fried foods, excessive exercise and certain medication raise the level of ROS in the body and aggravate the upheaval of oxidative stress. Antioxidants are potent enough for oxidative stress.

Antioxidants are the substances which are naturally produced within the body and are also available in countless foods items. They prevent cellular damage; improve immunity, eye health, neuromuscular system, and have regulatory effects on the body. The main antioxidants produced by the body are glutathione, lipoic acid, coenzyme Q 10 and N-acetyl Cysteine (NAC) and the enzymes like superoxide dismutase, catalase and glutathione peroxidase. Since other needed antioxidants cannot be synthesized in the body hence needs to be supplied by the food [1]. The family of antioxidants is comprised of vitamin $\mathrm{C}$ and $\mathrm{E}$, carotenoids; selenium, zinc, polyphenols, flavonoids and many other compounds found in green, purple, dark red and brown, yellow, orange and red coloured fruits and vegetables, nuts, berries, teas, spices, herbs and red meat, red wine. Essential oils also are high in antioxidant power.

Vitamin E quenches the lipid peroxyl radicals and prevents lipid peroxidation. Tocotrienol and tocochromanol, derivatives
Journal of

Nutrition and Health

\author{
Anjana Agarwal* \\ Nutritionist and Aromatherapist, SNDT Women's University, India \\ *Address for Correspondence \\ Anjana Agarwal, Nutritionist and Aromatherapist, SNDT Women's University, \\ Mumbai, India; E-mail: dranjanaagarwal@gmail.com \\ Submission: 11 September, 2017 \\ Accepted: 13 September, 2017 \\ Published: 25 September, 2017 \\ Copyright: $\odot 2017$ Agarwal A. This is an open access article distributed \\ under the Creative Commons Attribution License, which permits \\ unrestricted use, distribution, and reproduction in any medium, provided \\ the original work is properly cited.
}

of vitamin $\mathrm{E}$ at nanomolar concentration provide protection from neurodegeneration [2]. It has no activity against radicals in aqueous medium. Vitamin $\mathrm{C}$ is an electron donor and prevents other compounds to be oxidized. Lipoic acid prevents peroxidation and raises the intracellular glutathione levels and even counteracts the symptoms of deficiency of vitamin C and E. It can function both in lipid and aqueous phase; easily absorbed from the diet and crosses the blood-brain barrier. Hence it is used in the treatment of certain brain and neural disorders, diabetic neuropathy, cataract formation, $\mathrm{HIV}$ and radiation injuries $\mathrm{N}$-acetyl Cysteine (NAC) is a precursor of glutathione which itself is a strong antioxidant and helpful in DNA repair. It exhibits its biological effects against nitric oxide and improves the blood flow or acts as a vasodialator. Zinc is a co-factor of Superoxide Dismutase (SOD) and an inhibitor of NADPH oxidase and thus reduces the generation of ROS [3].

How will a person know the level of OS? It is easy. When a person is physically, mentally or emotionally exhausted, tired all day, frequently feeling pain in muscles and joints, having memory issues or brain fog, poor eye sight, grey hair or hair fall, susceptibility to noise, infection, he or she suffering from OS. Under these conditions, oxidation process is further aggravated by stress, anxiety, toxins and infections [4]. Addition of rich dose of antioxidant rich foods can mitigate many of the symptoms.

\section{References}

1. Kerksick C, Wiloughby D (2005) The antioxidant role of glutathione and $\mathrm{n}$-acetyl-cysteine supplements and exercise-induced oxidative stress. J Int Soc Sports Nutr 2 : 38-44

2. Packer L, Witt EH, Tritschler HJ (1995) alpha-Lipoic acid as a biological antioxidant. Free Radic Biol Med 19: 250-277.

3. Prasd AS (2014) Zinc is an antioxidant and anti-inflammatory agent: It's Role in human health. Front Nutr 1: 14

4. Lobo V, patil A, Phatak A, Chandra N (2010) Free radicals, antioxidants and functional foods: Impact on human health. Phamacogn Rev 4: 118-126. 